\title{
Type IV collagen degradation in the myocardial basement membrane after unloading of the failing heart by a left ventricular assist device
}

\author{
Annette H Bruggink ${ }^{1}$, Matthijs FM van Oosterhout ${ }^{1}$, Nicolaas de Jonge ${ }^{2}$, Jack PM Cleutjens ${ }^{3}$, Dick F van Wichen ${ }^{1}$,
} Joyce van Kuik ${ }^{1}$, Marcel GJ Tilanus ${ }^{1}$, Frits HJ Gmelig-Meyling ${ }^{4}$, Jan G van den Tweel ${ }^{1}$ and Roel A de Weger ${ }^{1}$

After left ventricular assist device (LVAD) support in patients with end-stage cardiomyopathy, cardiomyocytes decrease in size. We hypothesized that during this process, known as reverse remodeling, the basement membrane (BM), which is closely connected to, and forms the interface between the cardiomyocytes and the extracellular matrix, will be severely affected. Therefore, the changes in the myocardial BM in patients with end-stage heart failure before and after LVAD support were studied. The role of MMP-2 in this process was also investigated. Transmission electron microscopy showed that the BM thickness decreased post-LVAD compared to pre-LVAD. Immunohistochemistry indicated a reduced immunoreactivity for type IV collagen in the BM after LVAD support. Quantitative PCR showed a similar mRNA expression for type IV collagen pre- and post-LVAD. MMP-2 mRNA almost doubled post-LVAD $(P<0.01)$. In addition, active MMP-2 protein as identified by gelatin zymography and confirmed by Western blot analysis was detected after LVAD support and in controls, but not before LVAD support. Active MMP was localized in the BM of the cardiomyocyte, as detected by type IV collagen in situ zymography. Furthermore, in situ hybridization/immunohistochemical double staining showed that MMP-2 mRNA was expressed in cardiomyocytes, macrophages, T-cells and endothelial cells. Taken together, these findings show reduced type IV collagen content in the BM of cardiomyocytes after LVAD support. This reduction is at least in part the result of increased MMP-2 activity and not due to reduced synthesis of type IV collagen.

Laboratory Investigation (2007) 87, 1125-1137; doi:10.1038/labinvest.3700670; published online 17 September 2007

KEYWORDS: basement membrane; LVAD support; MMP-2; reverse remodeling; type IV collagen

Left ventricular assist devices (LVADs) are commonly used in patients with heart failure as a bridge to heart transplantation (HTx). In most cases, LVAD support extends the patient's lifespan and improves the quality of life. ${ }^{1,2}$ In addition, pressure and volume unloading of the left ventricle (LV) by LVAD can reverse left ventricular dilatation and leads to regression of left ventricular hypertrophy and neurohormonal changes. ${ }^{3-6}$ The understanding of this process, referred to as 'reverse remodeling," is important for a better insight into both myocardial events during LVAD support and the processes leading to heart failure. Additionally, several institutions described the possibility of LVAD explantation without the need for HTx (weaning). ${ }^{4,8-11}$ This requires a profound knowledge of the process of reverse remodeling.
In previous studies with cardiac unloading, we demonstrated partial recovery of the contractile myofilaments in the cardiomyocytes ${ }^{12}$ and decreased natriuretic peptide levels both in the plasma of the patients and in the heart. ${ }^{5,6}$ Since reverse remodeling not only involves the cardiomyocytes but also the extracellular matrix (ECM), we have focused on the changes in ECM before and after LVAD support. The ECM, which consists of the fibrillar collagens, type I and III collagen, and comprises a basement membrane (BM) surrounding cardiomyocytes, forms a continuum between different cell types within the myocardium and provides a structural supporting network to maintain myocardial geometry. ${ }^{13,14}$ We have shown that reverse remodeling of type I and III collagen in the ECM follows a biphasic pattern. ${ }^{15}$ Initially, an increase of type I and III collagen turnover took

\footnotetext{
'Department of Pathology, University Medical Center Utrecht, Utrecht, The Netherlands; ${ }^{2}$ Department of Cardiology, University Medical Center Utrecht, Utrecht, The Netherlands; ${ }^{3}$ Department of Pathology, University Maastricht, Maastricht, The Netherlands and ${ }^{4}$ Department of Immunology, University Medical Center Utrecht, Utrecht, The Netherlands

Correspondence: AH Bruggink, MSc, Department of Pathology H04.312, University Medical Center Utrecht, Heidelberglaan 100, PO Box 85500, Utrecht GA 3508, The Netherlands. E-mail: a.h.bruggink@umcutrecht.nll 
place, which was paralleled by a volume increase of the ECM. Subsequently, this turnover reduced and the ECM volume decreased, together with a restoration of the collagen network. Type IV collagen, which is one of the components of the $\mathrm{BM},{ }^{14}$ differs from the interstitial collagens by the presence of globular domains interspersed within the triplehelical segments. ${ }^{16}$ This unique feature gives type IV collagen the flexibility to assemble into a sheet like network. Therefore, type IV collagen in the BM links the sarcolemma of individual myocytes to the surrounding ECM and interstitial cells ${ }^{17}$ and is important in the transmission of force during diastole and systole. The function of laminin, another component of the BM, includes the mediation of adhesion, migration, growth and differentiation of cells. ${ }^{18-20}$

Degradation of the BM proteins occurs under a variety of physiological and pathological circumstances including embryogenesis, ${ }^{21}$ wound healing ${ }^{22}$ and metastasis ${ }^{23}$ by MMPs. It is known that MMP-2 is able to degrade type IV collagen and is upregulated during heart failure. ${ }^{14,24,25}$ Because we have shown changes in cardiomyocyte size and ECM volume after unloading of the LV by LVAD support ${ }^{12,15}$ and given the close connection between type IV collagen and the cardiomyocyte, we hypothesized that the BM surrounding the cardiomyocyte must be reshaped when the cardiomyocytes become smaller by unloading of the heart by an LVAD. If so, MMP-2 (collagenase IV) can play a major role in this process of remodeling. Therefore, in heart biopsies taken before and after LVAD support, the BM was studied with transmission electron microscopy (TEM), type IV collagen mRNA expression by quantitative RT-PCR (Q-PCR), and the presence of type IV collagen protein by immunohistochemistry (IHC). MMP-2 was studied by Q-PCR, in situ hybridization (ISH), IHC, Western blotting (WB), gel zymography and in situ zymography (ISZ) (localization and identification of active enzymes).

\section{MATERIALS AND METHODS Patients}

In this study, 25 patients with refractory end-stage heart failure were included. Characteristics of these patients are summarized in Table 1. All patients were treated with an LVAD (Heart-mate, Thoratec, Pleasanton, CA, USA) as a bridge to transplantation. Twenty-three of these patients were successfully transplanted. Two patients died before HTx; one as a result of recurrent cerebral embolism, the other due to chronic ischemic enteritis. All patients were in NYHA class IV at the time of LVAD implantation and in NYHA class I while on LVAD support. Informed consent to participate in this study was obtained from all patients. The myocardial biopsy at time of LVAD implantation consisted of the LV apical core removed during implantation. These biopsies (pre-LVAD) were compared with LV tissue specimens of the explanted hearts after HTx (post-LVAD) from the apical half of the LV, outside the suture area of the inflow cannula. In patients with IHD, care was taken to use myocardium away from the infarct zone, the 'unaffected' myocardium. In the two patients who died before transplantation, corresponding tissue specimens of the heart at autopsy were used. All biopsies were directly fixed in buffered formalin and embedded in paraffin. In addition, in 11 cases (pre- and post-LVAD) biopsies were frozen in liquid nitrogen after implanting the LVAD or after HTx, and in 10 cases (pre- and post-LVAD) biopsies were fixed in Karnovsky's fixative for TEM analysis. Control tissue was taken from the LV of nonused donor hearts $(n=6)$. From three of these hearts also samples were available for TEM evaluation.

\section{Transmission Electron Microscopy}

Heart tissue and biopsies of the LV were fixed in Karnovsky's fixative, followed by $4 \% \mathrm{OsO}_{4}$. After dehydration in alcohol, they were embedded in epon, following routine procedures.

Material was selected (unaware of the patient's background details) for longitudinal fiber localization in semithin sections, stained with toluidine blue and viewed by a light microscope. Ultrathin sections were stained with 5\% uranyl acetate and $2.5 \%$ lead citrate, randomly analyzed and photographically recorded under a TEM (Jeol 1200 EX-2). Measurement of the thickness of the BM was performed with Image J 1.25 (NIH, USA). Of all 10 patients and 3 control hearts, the BM of 10 cardiomyocytes was measured. Of each cardiomyocyte, the median of 12 measurements was taken and the mean of all 10 cardiomyocytes from one patient/ control was calculated.

TEM photographs (at least 17 for each patient) showing both collagen fibers and sarcolemma with a BM were analyzed. The number of photographs showing collagen but no contact with the BM and the photographs showing single contacts with the BM or showing bundles connected to the BM were counted and expressed as the $\%$ of all pictures.

For all analyses, the observer was blinded to the study group (pre- or post-LVAD or control) she studied.

\section{MMP Macro-Array and Q-PCR for Different MMP}

To characterize the gene expression profile of 23 different MMP in one RNA sample, RNA was isolated from frozen tissue sections using TRIzol ${ }^{\mathrm{TM}}$ Reagent (GibcoBRL, Rockville, MD, USA). Isolated RNA was directly used in the MMP GeArray kit (SuperArray, Frederick, MD, USA) following the GeArray User Manual. Differences in MMP and TIMP expression before and after LVAD support were calculated using the software included in the GeArray kit.

To confirm the MMP mRNA expression on the MMP GeArray, RNA was isolated from frozen myocardial tissue sections using TRIzol Reagent for Q-PCR. Three micrograms of RNA was used for cDNA synthesis using oligo-dT and random primers. Five microliters of a 1:30 dilution of the cDNA was used in the Assay-on-Demand kit for MMP-1, $-2,-8$, $-9,-13,-14,-15,-20,-25,-26$ and TIMP-1, -2, -3, -4 (Applied Biosystems, Foster City, CA, USA) for Q-PCR (Taqman P7700, Applied Biosystems). Porphobilinogen deaminase 
Table 1 Patients characteristics

\begin{tabular}{|c|c|c|c|c|c|}
\hline Patient & Gender & Age (years) & Disease & Days on LVAD support & Medication during LVAD support \\
\hline 1 & Female & 30 & DCM & 66 & Enalapril \\
\hline 3 & Male & 23 & DCM & 102 & Amlodipine \\
\hline 4 & Female & 27 & DCM & 124 & Enalapril \\
\hline 6 & Male & 19 & DCM & 178 & Amlodipine, Enalapril \\
\hline 7 & Female & 52 & DCM & 187 & None \\
\hline 8 & Male & 53 & DCM & 190 & None \\
\hline 9 & Male & 53 & DCM & 242 & Amlodipine, Enalapril \\
\hline 10 & Male & 23 & DCM & 297 & None \\
\hline 14 & Male & 18 & DCM & 455 & Amlodipine \\
\hline 15 & Male & 49 & IHD & 35 & None \\
\hline 16 & Male & 53 & IHD & 71 & Amlodipine \\
\hline 17 & Male & 42 & IHD & 72 & None \\
\hline 18 & Male & 28 & IHD & 97 & None \\
\hline 19 & Male & 48 & IHD & 106 & None \\
\hline 20 & Male & 52 & IHD & 113 & Amlodipine \\
\hline 21 & Male & 34 & IHD & 137 & Amlodipine \\
\hline
\end{tabular}

(PBGD) and $18 \mathrm{~S}$ were chosen as references. Total volume was $25 \mu$ l. The following thermo cycle profile was used for the MMP, PBGD and $18 \mathrm{~S}$ (housekeeping genes): $95^{\circ} \mathrm{C}$ for $10 \mathrm{~min}$ followed by 45 cycles of $95^{\circ} \mathrm{C}$ for $15 \mathrm{~s}$ and $60^{\circ} \mathrm{C}$ for $1 \mathrm{~min}$. Each sample was run in duplicate. The following formula was used to quantify the amount of mRNA in our samples: relative quantity $=2^{-\Delta \Delta C \mathrm{t}}, \Delta \Delta C_{\mathrm{t}}=\Delta C_{\mathrm{t}}$ (sample) $-\Delta C_{\mathrm{t}}$ (calibrator) and $\Delta C_{\mathrm{t}}=C_{\mathrm{t}}$ (target) $-C_{\mathrm{t}}$ (reference). The calibrator sample was cDNA from human placenta tissue, which was run with each experiment; therefore, it was possible to make an inter-run comparison.

\section{Q-PCR for Type IV Collagen $\alpha 1-\alpha 6$}

To determine the mRNA expression of the different $\alpha$-chains of type IV collagen in heart tissue, RNA was isolated from frozen tissue sections using TRIzol Reagent (GibcoBRL). Three micrograms of RNA was used for cDNA synthesis using oligo-dT and random primers. Five microliters of a 1:30 dilution of the cDNA was used in the Assay-on-Demand kits for all six $\alpha$-chains of type IV collagen (Applied Biosys- tems) in a Q-PCR assay (Taqman P7700, Applied Biosystems). PBGD was used as reference gene.

\section{MMP-2 RNA ISH and ISH/IHC Double Staining}

To identify cell types expressing MMP-2 mRNA, RNA ISH and RNA ISH/IHC double staining was performed as described by van Hoffen et al. ${ }^{26}$ MMP-2 and IL-2 were amplified using specific primers (MMP-2 forward: $5^{\prime}$-ATTCCGCTTCCAGGGCACATC- $3^{\prime}$ and MMP-2 reverse: $5^{\prime}$-GTTAAAGGCGGCACCACTCG-3'). The PCR products were labeled with digoxigenin, as described previously. The specificity of the probes for ISH was confirmed with tonsil as positive control. Specificity of the ISH for MMP-2 was further controlled by using a probe of similar composition and labeling directed against interleukin-2 (IL-2). ${ }^{26}$ Negative controls were obtained by overnight RNAse pretreatment of sections of heart tissue at $37^{\circ} \mathrm{C}$ (RNAse A $100 \mu \mathrm{g} / \mathrm{ml}$, RNAse $\mathrm{T} 1100 \mathrm{U} / \mathrm{ml}$, Boehringer-Mannheim $\mathrm{GmbH}$ ), and by omission of the probe from the hybridization mixture. For ISH/ IHC, double staining procedure was performed as described 
by Bruggink et al, ${ }^{5}$ using antibodies against CD3 (T cells), CD68 (macrophages) and factor 8 (endothelium) and the MMP-2 probe.

\section{IHC of Type IV Collagen, Laminin and MMP-2}

To localize type IV collagen, laminin and MMP-2 protein expression, a two-step immunohistochemical analysis was performed $^{12,27}$ on paraffin-embedded sections. Pepsin was used as antigen retrieval. Primary antibodies used were as follows: type IV collagen (polyclonal, 1:100; Novocastra Laboratories Ltd, Newcastle upon Tyne, UK), laminin (polyclonal, 1:200; Neomarkers, Fremont, USA) and MMP-2 (monoclonal, 1:20; Neomarkers). For type IV collagen, a second polyclonal antibody ${ }^{28}$ was used as second opinion. Subsequently, the slides were incubated with rabbit powervision (Klinipath, Duiven, The Netherlands). The specificity of the IHC was confirmed by positive and negative controls. Placenta was used as positive control. Omission of the primary antibody and replacement of the primary antibody by isotype-matched irrelevant antibodies served as negative controls.

\section{Western Blotting MMP-2}

Immunoblotting for MMP-2 was performed on proteins isolated from myocardial biopsies before and after LVAD support. Equal amounts of protein were separated on a $12 \%$ bis-Tris polyacrylamide gel (Bio-Rad Laboratories, Veenendaal, The Netherlands) and transferred to nitrocellulose membrane. WB analysis was performed with a rabbit polyclonal anti-MMP-2 (Santa Cruz Biotechnology, Inc., Santa Cruz, CA, USA) antibody (1:500) in TTBS containing $1 \%$ BSA. After incubation with horseradish peroxidase-conjugated secondary antibody, the blot was developed using Amersham ECL system (Amersham Pharmacia Biotech Europe $\mathrm{GmbH}$ ). $\alpha$-Actin was used as loading control. The intensity of the bands was quantified with the computer program TotallabV 2003.03 (Nonlinear Dynamics, Newcastle upon Tyne, UK).

\section{Gelatin Zymography}

Cardiac tissue was homogenized in $1 \times$ sample buffer containing $0.5 \%$ Triton X-100 (Sigma, Zwijndrecht, The Netherlands), $0.5 \mathrm{U} / \mathrm{ml}$ aprotinin (Sigma) and $0.01 \%$ sodium azide in PBS. The samples were centrifuged and the supernatants were collected and stored at $-20^{\circ} \mathrm{C}$ until use. MMP enzyme expression was assayed by SDS-PAGE zymography using gelatin as substrate. Equal volumes of samples were subjected to electrophoresis, without boiling or reduction, through an $8 \%$ polyacrylamide gel copolymerized with gelatin $(2 \mathrm{mg} / \mathrm{ml})$. After electrophoresis was completed, the gels were washed twice in $2.5 \%$ Triton X-100, rinsed in aquadest and incubated overnight in Brij 35 solution (50 mM Tris- $\mathrm{HCl}$ $\mathrm{pH} 7.4,10 \mathrm{mM} \mathrm{CACl}_{2}$ and $0.05 \%$ Brij-35). Finally, the gels were stained using Coomassie Blue (Sigma). Proteolytic bands were visualized by destaining the gels with $25 \% \mathrm{MeOH}$ and $15 \%$ acetic acid in MiliQ and quantified with the computer program TotallabV 2003.03.

\section{Type IV Collagen ISZ}

To demonstrate active MMP-2 in frozen heart tissue sections, ISZ was performed according to Rouet-Benzineb et al. ${ }^{29}$ Lysis of the substrate at the side of active MMP resulted in fluorescence, assessed by fluorescent microscopy or confocal laser scan microscopy. To test whether fluorescence was specific, some slides were preincubated with an MMP inhibitor (phenanthroline monohydrate $(20 \mu \mathrm{g} / \mathrm{ml}$; Sigma) in Tris- $\mathrm{HCl}$ ) and incubated with the substrate in combination with the inhibitor. All slides were examined by two investigators in a random blinded manner. The fluorescence was graded as follows: $0=$ negative; $1=$ weakly positive; $2=$ positive; $3=$ strongly positive; $4=$ very strongly positive. Values for each slide were obtained by taking the mean of all scored areas.

\section{Statistical Analysis}

Grading of the ISZ, Q-PCR data and BM measurements were compared using the paired Wilcoxon signed-rank test or the Mann-Whitney test. Inter- and intraobserver variability was tested using kappa analysis. All data were calculated with the statistical package of Prism 3.02. A $P$-value $<0.05$ was considered significant.

\section{RESULTS}

Since staining for type IV collagen, laminin and MMP showed no significant differences between patients with DCM and patients with IHD (excluding the infarcted areas), both patient groups are considered as one.

Given the difference in localization of the pre-LVAD myocardial biopsies (apex) and the post-LVAD biopsies (apical half of the LV), we examined whether regional differences were present in the heart by analyzing longitudinal sections ( 7 biopsies of LV, 4 of RV and 4 of septum) of several hearts. Using IHC (4 hearts) and ISZ (2 hearts), we did not observe grading differences between the various cardiac areas. The inter- and intraobserver kappa values for ISZ were 0.58 and 0.60 , respectively, and for TEM 0.77 and 0.77 , indicating fair agreement.

\section{Transmission Electron Microscopy}

LV biopsies of the apex (pre-LVAD) and LV wall (post-LVAD) were used for TEM ( $n=10$ patients). In biopsies taken preLVAD support, the BM of the cardiomyocytes was irregular in size and in most cases dispersed; the lamina lucida and lamina densa were difficult to discriminate. In biopsies taken after LVAD support, a clear lamina lucida and lamina densa of the BM was observed (Figure $1 \mathrm{~b}$ and $\mathrm{d}$ ). Two types of connections of the collagen fibers to the $\mathrm{BM}$ were discriminated: contacts of single collagen fibers or bundles of collagen fibers. In the biopsies taken before LVAD support, both single contacts as well as bundles of collagen fibers 

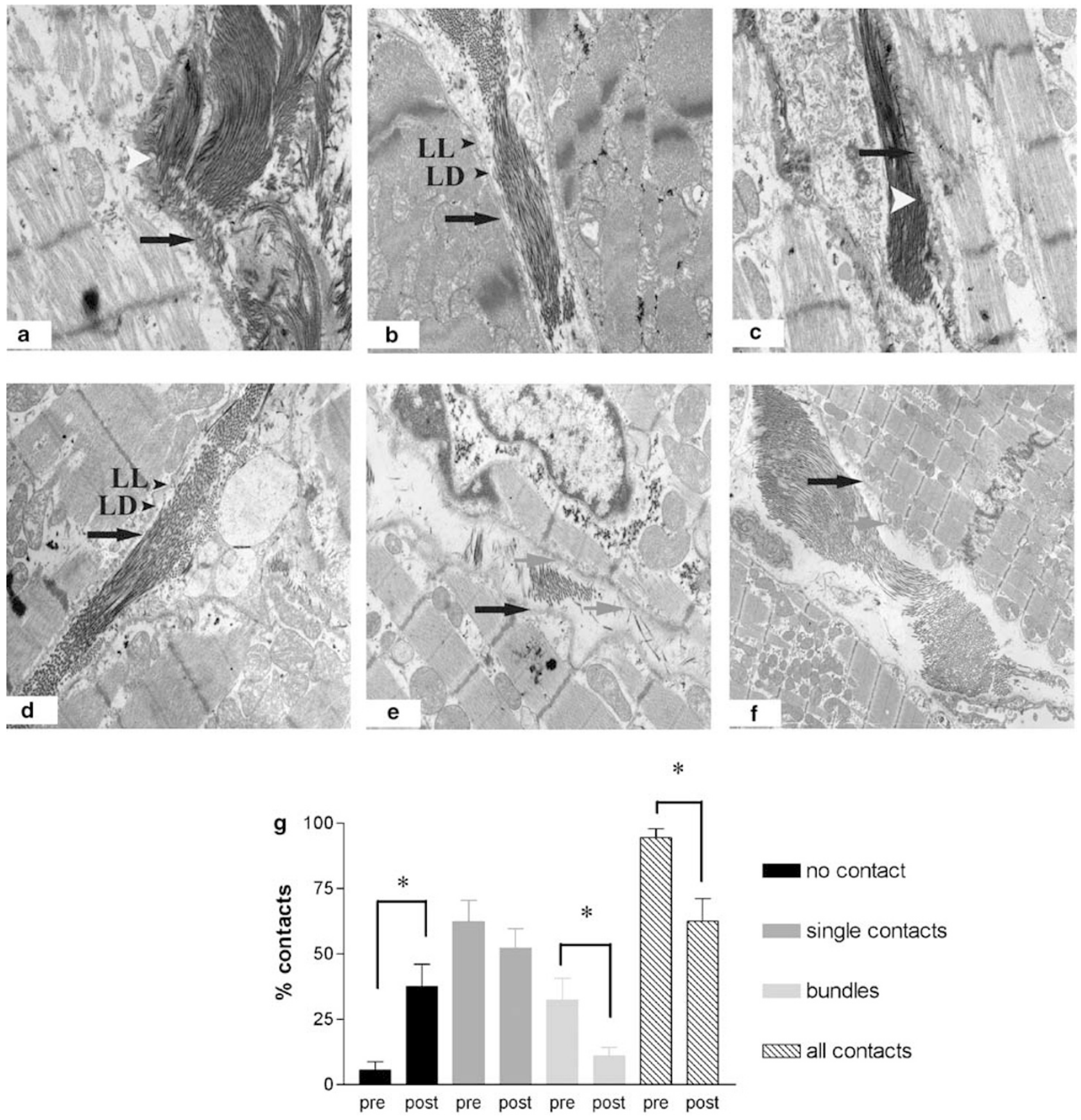

Figure 1 BM structure and thickness as observed with TEM before and after LVAD support. Representative examples ( $n=10)$ of TEM of heart biopsies. Figure shows two patients with end-stage heart failure, pre-LVAD support (a and $\mathbf{c}$ ) and post-LVAD support (b and d). (a) (12K) and (c) (10K): pre-LVAD, notice the irregular size of the BM and vague contours of the lamina lucida (LL) and lamina densa (LD) but also the tight connections of the ECM with the BM pre-LVAD. (b and d) Post-LVAD (both 10K), the LL and LD could be clearly distinguished but there was also a lack of connection of the ECM with the BM. (e) (10K) and (f) (4K): control $(n=3)$. Notice the irregular size of the BM and the clear contacts between the BM and the collagens fibers. Black arrow: BM; white arrowheads: bundles of collagen fibers connecting to the BM. Gray arrows: single collagen fibers connecting to the BM.

(g) Quantification of different contacts between collagen fibers in the ECM and the BM. Indicated is the \% of photographs showing collagen but no contact with the BM (no contact) and the photographs showing single contacts with the BM (single contacts in terms of magnification) or showing bundles connected to the $\mathrm{BM}$ (bundles). ${ }^{*} P<0.05 . \mathrm{K}=1000$ in terms of magnification.

connecting the BM were observed (Figure $1 \mathrm{a}, \mathrm{c}$ and $\mathrm{g}$ ). In contrast to the pre-LVAD biopsies, the connections of collagen bundles to the $\mathrm{BM}$ and the total number of contacts were significantly decreased after LVAD $(P<0.05$; Figure $1 \mathrm{~b}$, $\mathrm{d}$ and $\mathrm{g}$ ). In normal hearts, the BM showed the same irregular structure as the pre-LVAD BM. Also the collagen-BM contacts are similar between normal hearts and before LVAD (Figure 1e and f). The thickness of the BM decreased from 
$0.09 \pm 0.02 \mu \mathrm{m}$ pre-LVAD to $0.07 \pm 0.02 \mu \mathrm{m}(P=0.04)$ postLVAD support. The thickness of the BM in the healthy control group was $0.08 \pm 0.0006 \mu \mathrm{m}$ (Figure 1g).

\section{MMP Macro-Array and Q-PCR}

The MMP macro-array data showed that multiple MMP and TIMP were expressed in heart tissue $(n=11$; Figure $2 \mathrm{a}$ and $\mathrm{b})$ pre- and post-LVAD support. Moreover, some MMP displayed an altered expression after LVAD support: MMP-2 was significantly increased $(P=0.04)$ after LVAD support; however, MMP-24 decreased significantly $(P<0.01)$ after LVAD support.

Most of the array data were confirmed by Q-PCR. MMP-2 significantly increased both in the macro-array and by Q-PCR $(P<0.01$; Figure $2 \mathrm{c})$. However, MMP-24 did not change after LVAD support as shown with Q-PCR $(P=0.3)$. Furthermore, some MMPs, which were present in the macroarray, were almost not detectable in Q-PCR (MMP-13, MMP-20, MMP-26). The reason for the discrepancies was not clear. We considered the Q-PCR data more specific, as most MMPs detected by the array were just above the detection limit and some cross reactivity could not be excluded of the arrays.

\section{Type IV Collagen $\alpha 1-\alpha 6$ mRNA Expression in the Heart} Q-PCR for type IV collagen $\alpha 1-\alpha 6$ was performed using mRNA as templates from frozen heart biopsies, from patients with end-stage heart failure pre- and post-LVAD $(n=11)$ and biopsies from control hearts $(n=6$; Table 2$)$. For all $\alpha 1-\alpha 6$ chains of type IV collagen, mRNA was present in the heart. There was no significant difference between expression levels of the six $\alpha$-chains before and after LVAD support. However, although not statistically significant, mRNA of the major collagen $\alpha 3$ and $\alpha 4$ chains tended to increase after LVAD support. Compared to the control group, after LVAD the mRNA expression of the $\alpha 3$ chain was two times higher $(P=0.02)$, the expression of the $\alpha 4$ chain was significantly decreased, both in the pre- and post-LVAD support groups, and the expression of the minor $\alpha 6$ chain was also decreased before LVAD compared to the control group $(P<0.01$; Table 2).

\section{Type IV Collagen and Laminin Protein Expression before and after LVAD Support}

Formalin-fixed biopsies from 25 patients before and after LVAD support were immunohistochemically stained for type IV collagen and laminin, both principal components of the BM.

\section{Type IV collagen, IHC}

Using two different antibodies, in all biopsies taken before LVAD support (Figure 3a) type IV collagen was abundantly present in the BM surrounding the cardiomyocyte and underneath the endothelial layer of blood vessels. All the biopsies taken after LVAD support showed a strong reduction or complete disappearance of staining of the BM surrounding the cardiomyocytes, for both type IV collagen antibodies tested, while the BM of the blood vessels remained positive for type IV collagen (Figure 3b).

\section{Laminin}

Immunoreactivity of laminin was strongly present in the BM surrounding the cardiomyocytes in all biopsies taken before and after LVAD support. After LVAD support, this immunoreactivity did not change significantly (Figure $3 \mathrm{c}$ and $\mathrm{d})$.

\section{MMP-2 mRNA and Protein Expression in the Heart during LVAD Support \\ Quantitative PCR}

MMP-2 mRNA expression was almost doubled post-LVAD compared to pre-LVAD $(P=0.01, n=11)$ as shown by Q-PCR. Furthermore, the MMP-2 mRNA expression showed three and six times higher levels compared to the control group $(P<0.01$ and 0.001 , respectively), in pre- and postLVAD, respectively (Table 2 ).

\section{In situ hybridization}

With MMP-2 mRNA ISH $(n=11)$, a weak staining in the sarcoplasma of the cardiomyocytes was observed. In the ECM, the infiltrating cells and the endothelial cells in the blood vessels showed a positive staining for MMP-2 mRNA (Figure 4a), the other ECM components were negative.

To show MMP specificity, an IL-2 probe was used. As shown in Figure $4 \mathrm{~b}$, this probe stained a few lymphocytes present in the heart and not cardiomyocytes and endothelium as the MMP-2 probe did. Omitting the MMP-2 probe in the procedure, or RNAse treatment of the slides resulted in absence of positive staining.

ISH/IHC double staining was performed using monoclonal antibodies for CD3 (T cells), CD68 (macrophages) and factor 8 (endothelium), in combination with an MMP-2labeled probe.

MMP-2/CD68: There was a considerable amount of macrophages present in the myocardium, and almost all of these macrophages showed mRNA expression for MMP-2 (Figure 4c).

$M M P-2 / C D 3:$ In the myocardium, only few $\mathrm{T}$ cells were present both pre- and post-LVAD, and about half of these T cells were positive for MMP-2 mRNA (Figure 4d).

MMP-2/factor 8: MMP-2 mRNA was abundantly present in factor 8-positive endothelial cells in the myocardial capillaries and blood vessels (Figure 4e). With both techniques (ISH and ISH/IHC double staining), no significant differences in MMP-2 expression and localization were observed between LV biopsies before and after LVAD support (data not shown).

\section{Immunohistochemistry}

Cardiomyocytes pre- and post-LVAD support expressed sarcoplasmic staining for MMP-2 by IHC ( $n=25$; Figure $4 \mathrm{f})$. 
a

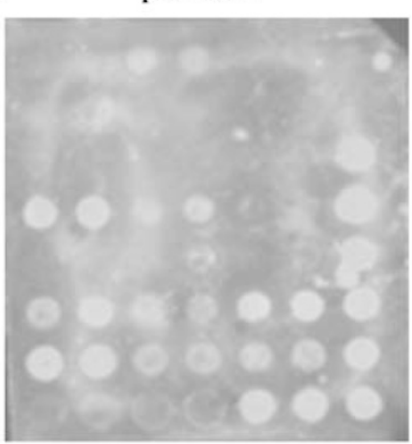

b
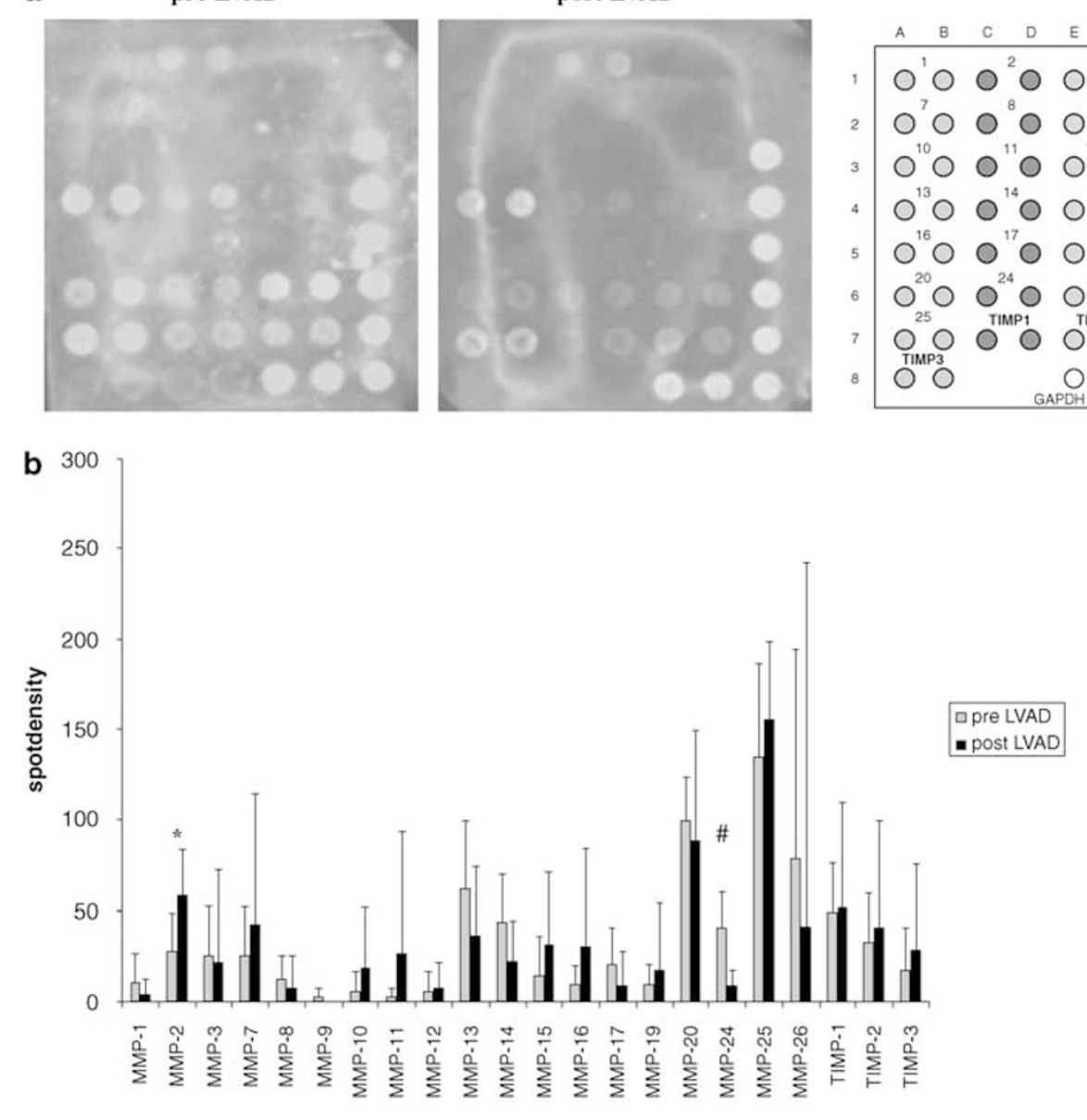
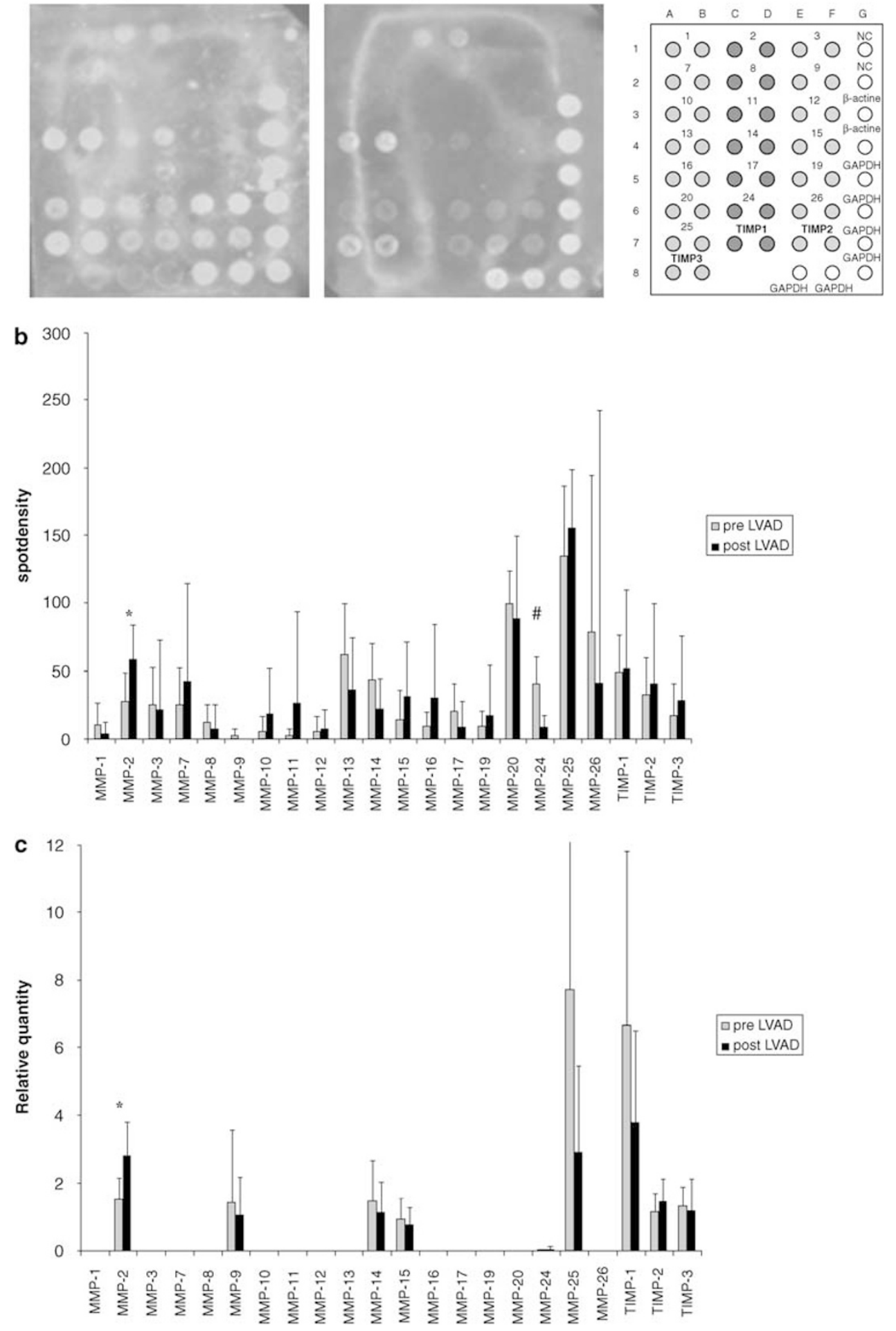

Figure 2 Macro-array and Q-PCR data for MMP and TIMP expression in pre- and post-LVAD heart tissue. (a) Representative example of pre- and post-LVAD macro-array analysis. Right panel shows the distribution of the various MMPs and TIMPs on the macro-array. Numbers correspond with the respective MMP. Also control spots are indicated. (b) Quantification of macro-array data on expression of MMP and TIMP pre- and post-LVAD $(n=11)$. ${ }^{\star} P=0.04$ pre- vs post-LVAD, ${ }^{\#} P<0.01$ pre- vs post-LVAD. (c) Q-PCR data of the expression of MMP and TIMP pre- and post-LVAD ( $\left.n=11\right)$. All indicated MMP and TIMP were tested, so bar present indicates no expression detected. ${ }^{\star} P<0.01$ pre- $v$ s post-LVAD. 


\section{Table 2 Quantitative RT-PCR for all the different $\alpha$-chains of type IV collagen and MMP-2}

\begin{tabular}{cccc}
\hline mRNA & Pre-LVAD & Post-LVAD & Control \\
\hline Type IV collagen & & & \\
$\alpha 1$ & $0.6 \pm 0.4$ & $0.6 \pm 0.6$ & $1.2 \pm 0.6$ \\
$\alpha 2$ & $0.2 \pm 0.2$ & $0.2 \pm 0.2$ & $0.2 \pm 0.1$ \\
$\alpha 3$ & $13.0 \pm 9.0$ & $21 \pm 12^{\#}$ & $9.4 \pm 8.8$ \\
$\alpha 4$ & $39.0 \pm 36^{\#}$ & $52 \pm 37^{\#}$ & $99.1 \pm 34.4$ \\
$\alpha 5$ & $0.6 \pm 0.5$ & $1.0 \pm 0.7$ & $0.8 \pm 0.3$ \\
$\alpha 6$ & $1.7 \pm 1.5^{\#}$ & $2.4 \pm 1.7$ & $2.7 \pm 0.5$ \\
MMP-2 & $1.3 \pm 0.7^{\#}$ & $2.5 \pm 0.8^{*, \#}$ & $0.4 \pm 0.3$
\end{tabular}

Relative abundance of mRNA as determined by Q-PCR of the different $\alpha$-chains of type IV collagen and of MMP-2 in snap-frozen myocardial biopsies from patients $(n=11)$ with end-stage heart failure taken before (pre-) LVAD and at time of heart transplantation (post-LVAD), compared with biopsies taken from unused donor hearts (control; $n=6$ ).

Data are presented as the mean value \pm s.d. (relative abundance).

${ }^{*} P<0.01$ pre-LVAD vs post-LVAD; ${ }^{\#} P<0.01$ pre-LVAD and/or post-LVAD vs control.

The cardiomyocytes located in the trabecula and near the epicardium and endocardium stained stronger than the cardiomyocytes localized in the center of the biopsy. In the ECM, some fibrotic areas, fibroblasts, capillaries and infiltrating cells stained positive. In some cases, the endothelial cells in the blood vessels and the coronary arteries were positive.

\section{Increased MMP-2 Activity after LVAD Support}

To quantify the MMP-2 protein expression in the heart, proteins isolated from heart tissue of 11 patients pre- and post-LVAD and 4 healthy controls were analyzed by WB. Total MMP-2 did not change pre- $v s$ post-LVAD. However, the active form of MMP-2 (represented by the $62 \mathrm{kDa}$ band) was significantly lower pre-LVAD compared to post-LVAD and the healthy control $(P=0.04$ and 0.01 , respectively; Figure 5a). After LVAD, the MMP-2 levels returned to normal. The inactive form (as represented by the $72 \mathrm{kDa}$ band) did not change significantly.

Gelatin zymography was performed on extracts from frozen heart biopsies $(n=11)$. One band was detected in all pre-LVAD samples and two bands were detected post-LVAD (Figure $5 \mathrm{~b}$ ). In the pre-LVAD sample, the $72 \mathrm{kDa}$ band was generated by inactive MMP-2. After LVAD, beside the inactive band an additional band, generated by active MMP-2 $(62 \mathrm{kDa})$ was present. Quantification of the 72 and $62 \mathrm{kDa}$ bands showed a significant increase of both the inactive and the active form of MMP-2 post-LVAD $(P<0.001)$ compared to pre-LVAD. In normal heart tissue, active MMP was also present as shown by the $62 \mathrm{kDa}$ band. However, active MMP2 was significantly higher after LVAD than in the healthy control $(P<0.01)$.

\section{Active MMP is Localized in the BM}

Active MMP was localized using type IV collagen ISZ in frozen tissue sections from 11 patient's pre- and post-LVAD implantation. Type IV collagen ISZ showed in both pre- and post-LVAD samples a collagenolytic activity, localized in or near the BM, surrounding the cardiomyocyte and in the sarcoplasm of the cardiomyocyte (Figure 6). The ECM showed no or only weak MMP activity. Semiquantitative grading of collagenolytic activity using a $0-4$ grading scale did not show significant differences in membrane bound ( $2.5 \pm 0.8 v s \quad 2.3 \pm 0.5$, NS) and sarcoplasmic (1.8 \pm 0.6 and $2.0 \pm 0.4$, NS) activity in pre- $v s$ post-LVAD sample, respectively. However, ISZ is not a quantitative method, and mainly shows the localization of active MMP. After incubation of the tissue sections with an MMP inhibitor, no fluorescent signal was observed (data not shown), indicating that the collagenolytic signal was MMP specific.

\section{DISCUSSION}

Left ventricular force production is dependent on the coupling of myocytes to the ECM, which is mediated through the $\mathrm{BM}$. We hypothesized that in patients with end-stage heart failure reverse remodeling during unloading of the LV by an LVAD, consisting among others of reversal of cardiomyocyte hypertrophy, would be associated with remodeling of the BM. The present study indeed demonstrates post-LVAD support structural alterations in the BM surrounding the cardiomyocytes, which was illustrated best by the decreased immunoreactivity for type IV collagen. Type IV collagen mRNA did not alter during LVAD support, indicating that the decreased collagen was not due to a decreased synthesis. However, we detected increased MMP-2 mRNA and MMP-2 protein levels and MMP-2 activity as assessed by the presence of a $62 \mathrm{kDa}$ band of active MMP in gel zymography and WB after LVAD support, suggesting an increased degradation of type IV collagen. Type IV collagen ISZ indicated that the active MMP was located within the BM of the cardiomyocyte.

The novel finding in this respect is that after LVAD support most parameters return to a more 'normal' situation. However, the BM clearly shows an exception to this rule.

The use of LVADs in end-stage heart failure has increased over the last two decades, since these devices became generally available. ${ }^{30}$ The majority of the LVADs have been used as a bridge to transplantation; however, in some cases successful explantation of the LVAD was reported. ${ }^{4,8-11}$ Unloading of the heart by an LVAD leads to reversal of LV dilatation and to regression of LV myocyte hypertrophy. ${ }^{1,3,12,31}$ The mechanisms of this reverse remodeling process are still largely unknown; therefore, the analysis of changes in the heart pre- and post-LVAD is important. The ECM, which consists of fibrillar collagens (type I and type III collagens) and a BM, forms a continuum between different cell types within the myocardium and provides a structural supporting network to maintain myocardial geometry. 

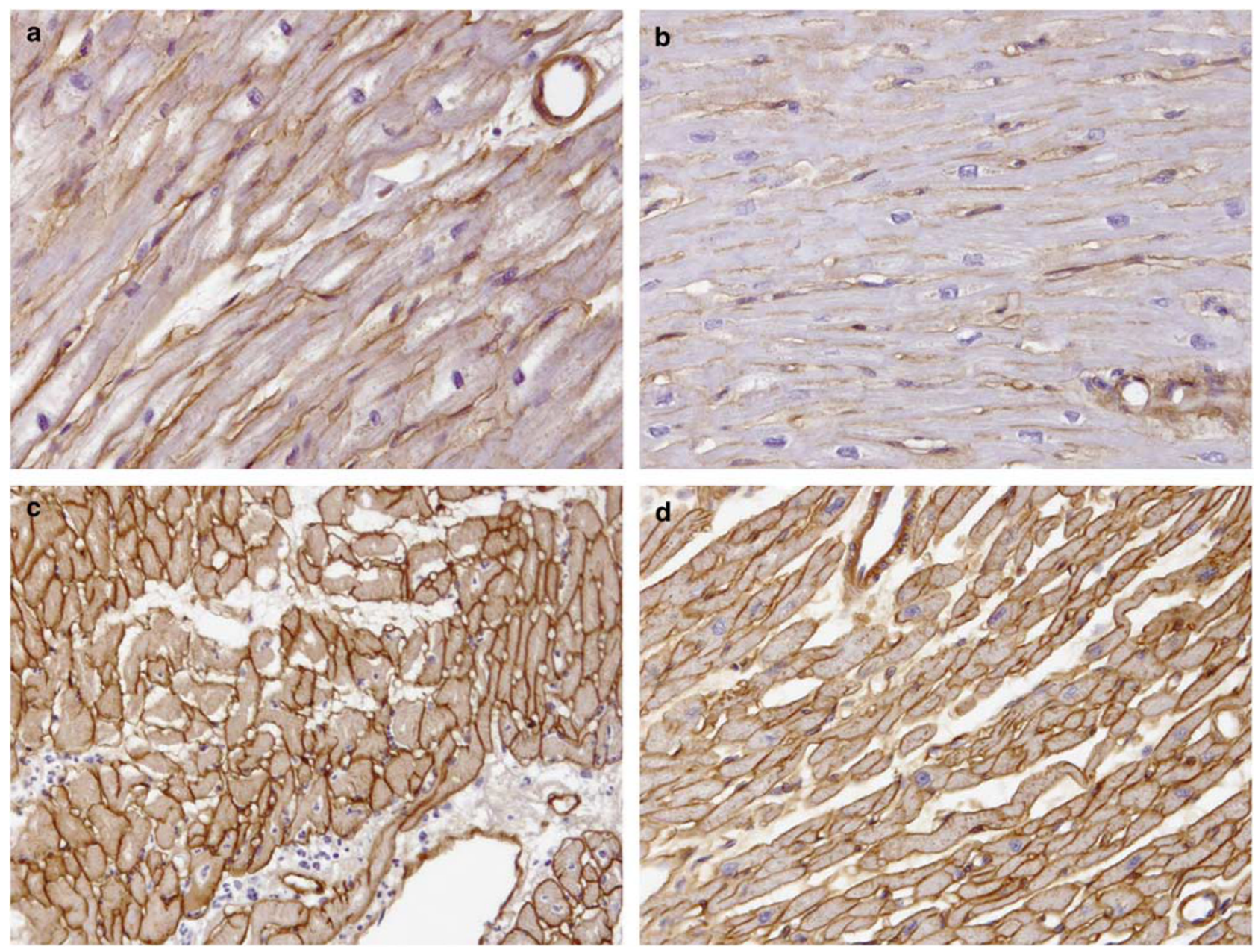

Figure 3 Type IV collagen and laminin immunohistochemical staining of patients with end-stage heart failure pre- and post-LVAD support. (a) Immunohistochemical staining of type IV collagen pre-LVAD $(\times 200)$. (b) A clear and consistent decrease of type IV collagen immunoreactivity was seen after post-LVAD ( $\times 200)$. (c) Immunohistochemical staining of laminin pre-LVAD $(\times 200)$. (d) After LVAD, no differences in staining pattern of laminin was detected compared to pre-LVAD $(\times 200)$.

Several groups have studied the changes of the fibrillar collagens in the ECM in heart failure ${ }^{32-34}$ and ECM after LVAD support. ${ }^{35-37}$ In a previous study of our group, ${ }^{15}$ we have shown that reverse remodeling of type I and III collagen in the ECM follows a biphasic pattern. Initially, an increase of type I and III collagen turnover takes place, which is paralleled by an increase of the ECM volume. Subsequently, after a longer period of LVAD therapy this turnover reduces and the ECM volume decreases, together with a restoration of the collagen network. We hypothesized that the BM and, therefore, type IV collagen and laminin must be remodeled during LVAD support because of the decreased cardiomyocyte size after LVAD support. This decrease in size was not biphasic, but reached its end point within the first 100 days on LVAD support. ${ }^{15}$ Indeed, LVAD support changed the morphology of the BM as assessed by TEM. The pre-LVAD biopsies from hearts with end-stage heart failure showed a thickened and irregular BM along the cardiomyocyte, and there were close connections between the collagen fibers in the ECM and the
BM. Normal heart tissue showed a similar morphology. After LVAD, the BM became more regular and the lamina lucida and lamina densa were compacter and could be clearly discerned. However, a disturbed connection between the BM and the collagen fibers in the ECM was observed. Although the exact consequences of these structural alterations are not known, it seems that connections between the collagen fibers and BM benefit or are more easily formed when the BM has a more irregular and thick structure. Interestingly, in contrast to most parameters showing normalization after LVAD support, the BM in normal hearts compares better with the preLVAD than the post-LVAD situation. These post-LVAD alterations may contribute to the reduced force generation of the heart. The differences in this association between the $\mathrm{BM}$ and collagen fibers may be due to alterations of the BM but may also be caused by the changes in the ECM. ${ }^{15,36,37}$

With IHC, we showed a significant reduction of type IV collagen immunoreactivity in the BM after LVAD support. However, laminin was still present in the BM after unloading 

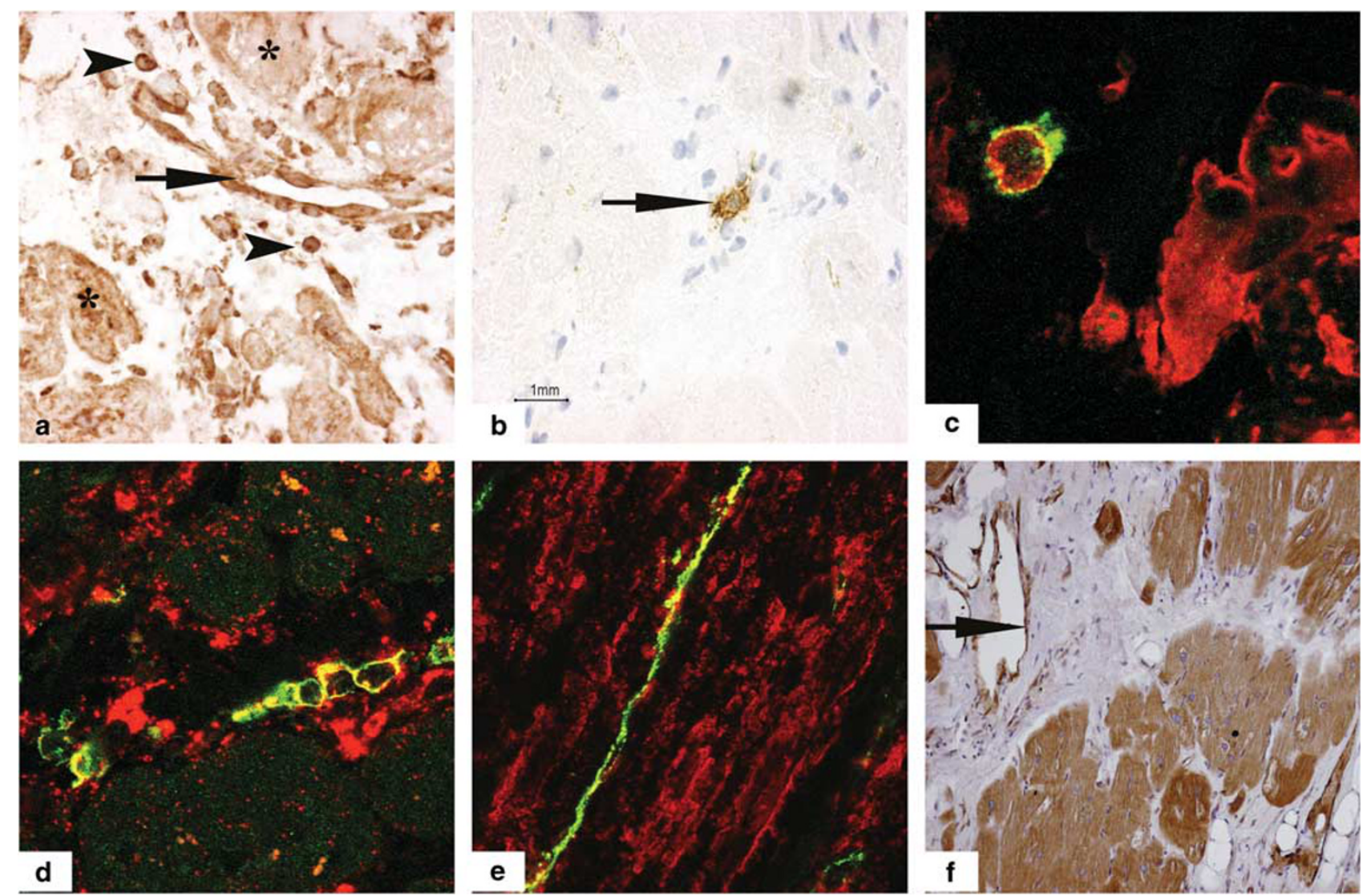

Figure 4 MMP-2 mRNA expression sites by ISH in the myocardium of patients with end-stage heart failure. Representative examples $(n=11)$ of MMP-2 mRNA expression in myocardial biopsies were taken before LVAD support. No significant change in expression was observed after LVAD support (data not shown). (a) Overview of MMP-2 ISH ( $\times 200$ ). MMP-2 mRNA expression by endothelial cells of the blood vessel (arrow), infiltrating cells (arrowhead) and the cardiomyocytes (asterisk). (b) Overview of IL-2 ISH ( $\times 200$ ). IL-2 expression by infiltrating cells in the heart (arrow). No staining was observed in the cardiomyocytes and endothelium. (c) Overlay of MMP-2 ISH-CD68 double staining $(\times 200)$. MMP-2 is in red and CD68 in green. CD68-positive macrophages expressed MMP-2 mRNA as was shown by the yellow color indicating MMP-2 and CD68 double staining. (d) Overlay of MMP-2 ISH-CD3 double staining ( $\times 50$ ). MMP-2 is in red and CD3 in green. CD3-positive T lymphocytes expressed MMP-2 mRNA as was shown by the yellow color indicating MMP-2 and CD3 double staining. (e) Overlay of MMP-2 ISH-factor 8 double staining $(\times 50)$. MMP-2 is in red and factor 8 in green. Factor 8 -positive endothelium expressed MMP-2 mRNA as was shown by the yellow color indicating MMP-2 and factor 8 double staining. (f) MMP-2 protein expression, as indicated by immunoreactivity in representative myocardial biopsy taken before LVAD support $(\times 200, n=25)$. Comparable results were obtained by ISH-IHC (double) staining as shown in (b-d). No significant change in staining pattern intensity was observed after LVAD implantation (data not shown). Arrow = endothelial cells of a blood vessel.

of the heart. This suggested that although the BM still surrounds the cardiomyocytes, its structure is severely disorganized. With Q-PCR, type IV collagen $\alpha 1-\alpha 6$ mRNA expression showed no significant difference before and after LVAD support. Compared to the control group type IV collagen $\alpha 4$ mRNA both pre- and post-LVAD was significantly reduced. Furthermore, compared to the control group a significant increase of the $\alpha 3$ chain and a significant decrease of the $\alpha 6$ chain were observed. So, in heart failure and after LVAD support the composition of the BM may be slightly different from the normal BM. However, our data indicated that the type IV collagen reduction after LVAD support is not due to a completely reduced synthesis of type IV collagen and must at least in part be the result of an increased degradation by MMP activity. To find out whether MMPs were involved, we investigated 23 different MMP and
TIMP in heart biopsies from eight patients before and after LVAD support with an MMP macro-array and confirmed these measurements with Q-PCR. From these array studies, only MMP-2 showed a significant increased $(P<0.01)$ expression after LVAD support. Others have found also other MMPs to be involved in the process of reverse remodeling, that is, MMP- $9,{ }^{36}$ but these could not be confirmed in this study. It is known that MMP-2 is able to degrade type IV collagen, but not laminin, in the $\mathrm{BM}^{33,34,38}$ Falk et $a^{33}$ has demonstrated a correlation between the upregulation of MMP-2 and the degradation of the BM. So, MMP-2 is able to degrade type IV collagen and therefore can play a major role in remodeling of the BM. The role of MMP-2 before and after LVAD support was therefore studied in more detail. First the MMP production sites were investigated. With MMP-2 ISH/ IHC, we showed that $\mathrm{CD}^{+}$cells, macrophages and the 
a

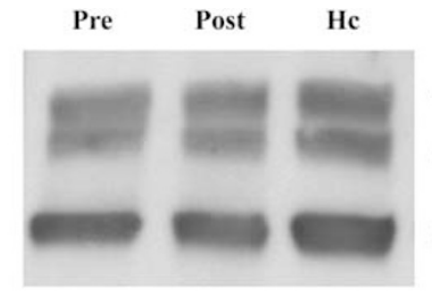

$72 \mathrm{KDa}$

$62 \mathrm{KDa}$

loading control

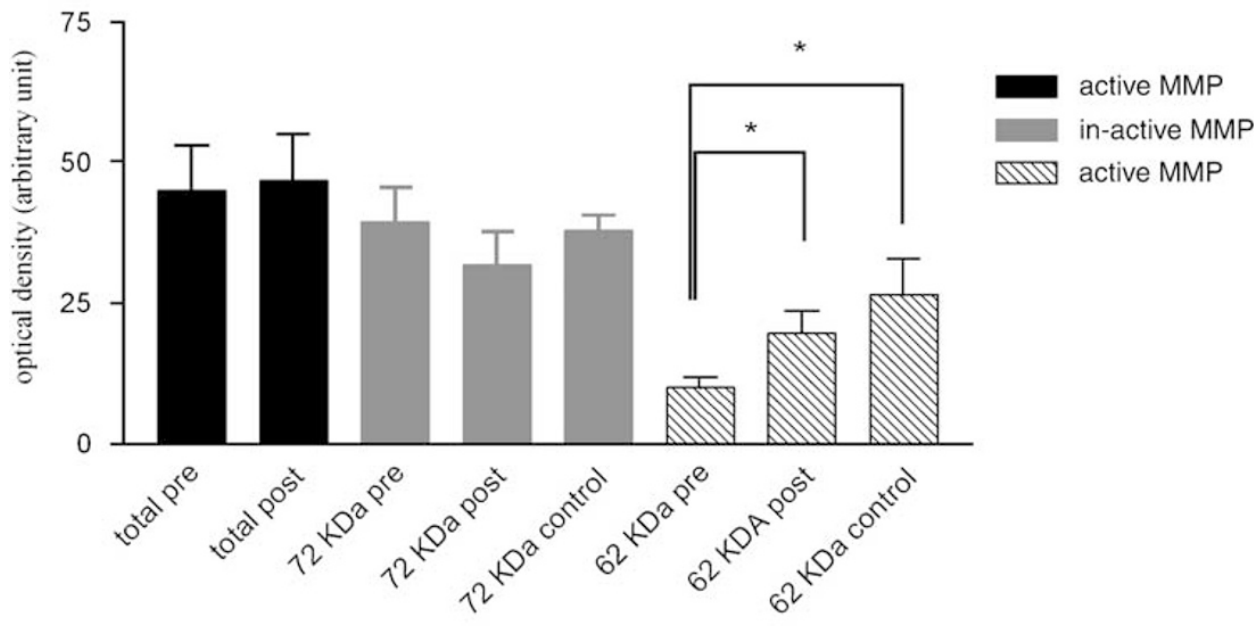

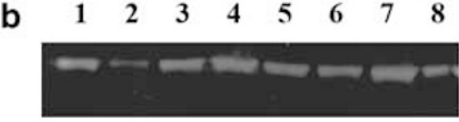

pre

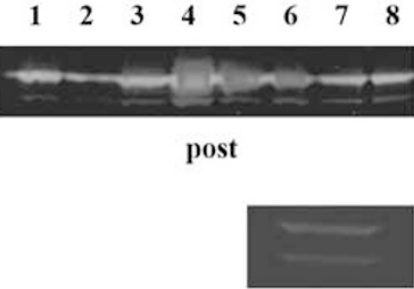

pat nr.

$72 \mathrm{kDa}$

$62 \mathrm{kDa}$

$72 \mathrm{kDa}$

$62 \mathrm{kDa}$

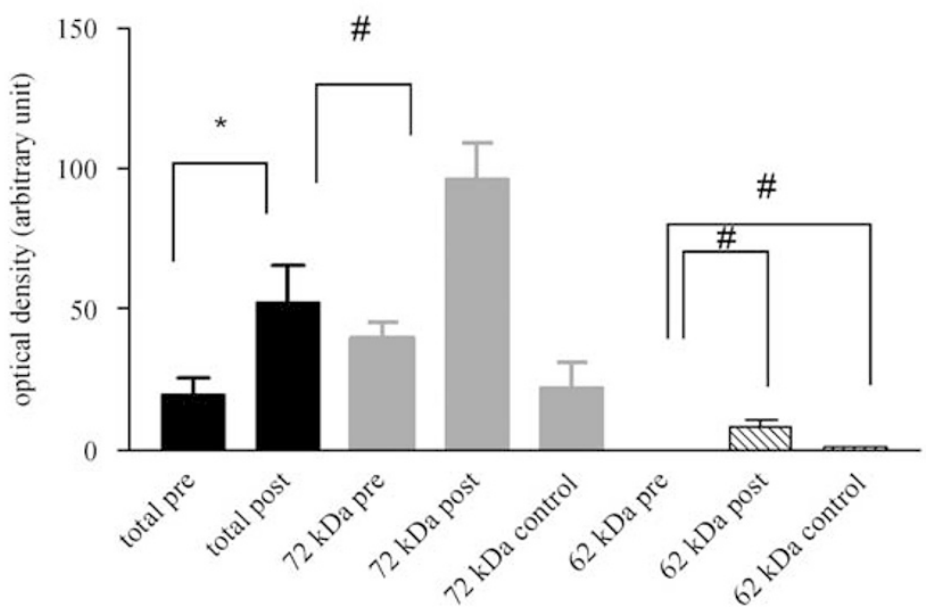

Figure 5 MMP-2 activity pre- and post-LVAD by WB and gelatin zymography. (a) A representative immunoblot $(n=11)$ stained with anti-MMP-2 in myocardial biopsies pre- and post-LVAD support and controls $(n=6)$. The $72 \mathrm{kDa}$ band represents inactive MMP-2 and the $62 \mathrm{kDa}$ band represents active MMP-2. Quantification of the immunoblot, as presented in the bar graph, showed significant increase of active MMP-2 after LVAD support ( $\left.{ }^{*} P=0.04\right)$. (b) Example of gelatin zymography gels showing MMP zymographic activity in 8 of 10 pre- and post-LVAD samples. Inactive MMP-2 is represented by the $72 \mathrm{kDa}$ band. Remarkably, the active MMP-2 (represented by the $62 \mathrm{kDa}$ band) was only present in the post-LVAD and control samples. The bar graphs show quantification of the zymographic activity. There was a significant increase in total MMP and in both the inactive and active form of MMP-2 after LVAD support as compared to pre-LVAD and the healthy controls $\left({ }^{*} P<0.0001,{ }^{\#} P<0.01\right)$. 

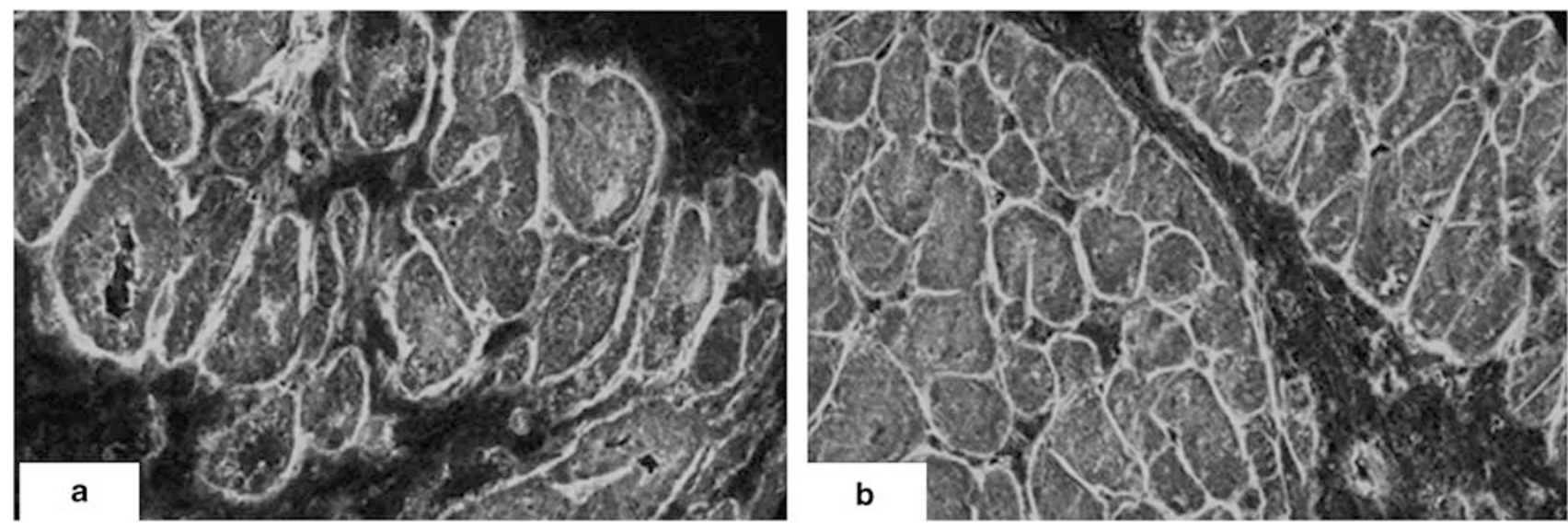

Figure 6 Type IV collagen ISZ pre- and post-LVAD support. Representative examples $(n=11)$ of ISZs of frozen biopsies pre-LVAD (a) and post-LVAD (b) $(\times 400)$. Collagenolytic activity was prominently present in or near the BM of cardiomyocytes and to a lesser extend in the sarcoplasm. The ECM showed mostly weak activity. The pattern of staining was not different before and after LVAD. Signal specificity was confirmed by preincubation with an MMP inhibitor (data not shown).

endothelium of blood vessels stained positive for MMP-2 mRNA. IHC studies also indicated that MMP-2 protein was located in the sarcoplasm of the cardiomyocytes, infiltrating cells and the endothelium of blood vessels. This indicated that MMP-2 can be produced in the heart by various cell types.

MMP-2 WB showed a low level of active MMP-2 protein pre-LVAD compared to the healthy control. After LVAD support, the level of active MMP returns to normal. Gel zymography showed some MMP-2 activity in normal hearts. However, after LVAD support MMP activity was significantly increased compared to pre-LVAD. So, both $\mathrm{WB}$ and gel zymography showed an increase in active MMP. This change in active MMP is confirmed by others in heart failure ${ }^{39,40}$ and during LVAD support ${ }^{36}$ by gel zymography. WB and gel zymography differed in the detected amount of inactive MMP. This may be explained by the difference in sensitivity of the methods, as described by Masure et al $^{41}$ and Descamps et $a l .{ }^{42}$ In normal hearts, active MMP was present and it is suggested that this is necessary for a normal collagen turnover in the heart. However, the detected increased levels of active MMP post-LVAD can lead to alterations in the BM membrane. Type IV collagen ISZ showed that this active MMP was present in the BM of the cardiomyocyte before and after LVAD support. Combining the gel zymography and ISZ data, it is likely that the active MMP-2 is at least in part responsible for the degradation of the BM during LVAD support, although we cannot exclude that other enzymes are involved in the BM breakdown as well.

Although there was a wide variation in the duration of LVAD support (35-557 days), no differences in the reduction of type IV collagen immunoreactivity and MMP-2 localization or activity have been detected between patients supported for a short period or for a long period of time. This suggests that the type IV collagen breakdown in the BM by
MMP occurs in the first period after LVAD support and the heart is not able to restore the BM properly. These findings support our previous results, which indicated that the decrease in size of the cardiomyocytes occurred within the first 100 days after LVAD implantation. ${ }^{15}$

In conclusion, LVAD support leads to reverse remodeling, including the decrease of cardiomyocyte size. Although the BM becomes more regular after LVAD support, as seen by TEM, type IV collagen is not or only weakly detectable in the BM, probably due to increased MMP-2 activity. Furthermore, disturbed connections between the BM and the collagen fibers in the ECM were observed by TEM after LVAD support. The disturbed linkage between the BM and the collagen fibers in the ECM may lead to impaired force transmission. This could be one of the reasons why weaning of the LVAD in patients with severe heart failure is only possible in a minority of patients. It may be speculated that restructuring of the BM requires the stimulus of cardiac reloading.

\section{ACKNOWLEDGEMENT}

We thank Dr M Vos and Dr A Groenewegen from the Department of Physiology, UMC Utrecht, for their kind gift of heart tissue of six non-used donor hearts. We also thank Dr D Shvartz from the research lab of the Department of Pathology, UMC Utrecht, for his technical support with WB. Technical assistance of Mrs P Homoet and Mrs P van der Weide is highly appreciated. This study was supported by the Netherlands Heart Foundation, NHF Grant 2004T31.

1. de Jonge $\mathrm{N}$, Kirkels $\mathrm{H}$, Lahpor JR, et al. Exercise performance in patients with end-stage heart failure after implantation of a left ventricular assist device and after heart transplantation: an outlook for permanent assisting? J Am Coll Cardiol 2001;37:1794-1799.

2. Grady KL, Meyer PM, Mattea A, et al. Change in quality of life from before to after discharge following left ventricular assist device implantation. J Heart Lung Transplant 2003;22:322-333.

3. Estrada-Quintero T, Uretsky BF, Murali $\mathrm{S}$, et al. Neurohormonal activation and exercise function in patients with severe heart failure and patients with left ventricular assist system. A comparative study. Chest 1995;107:1499-1503. 
4. Frazier $\mathrm{OH}$, Myers TJ. Left ventricular assist system as a bridge to myocardial recovery. Ann Thorac Surg 1999;68:734-741.

5. Bruggink $A H$, de Jonge $\mathrm{N}$, van Oosterhout MF, et al. Brain natriuretic peptide is produced both by cardiomyocytes and cells infiltrating the heart in patients with severe heart failure supported by a left ventricular assist device. J Heart Lung Transplant 2006;25:174-180.

6. Kemperman $\mathrm{H}$, van den Berg $\mathrm{M}$, Kirkels $\mathrm{H}$, et al. B-type natriuretic peptide (BNP) and N-terminal proBNP in patients with end-stage heart failure supported by a left ventricular assist device. Clin Chem 2004;50:1670-1672.

7. Levin HR, Oz MC, Chen JM, et al. Reversal of chronic ventricular dilation in patients with end-stage cardiomyopathy by prolonged mechanical unloading. Circulation 1995;91:2717-2720.

8. Hetzer R, Muller J, Weng $\mathrm{Y}$, et al. Cardiac recovery in dilated cardiomyopathy by unloading with a left ventricular assist device. Ann Thorac Surg 1999;68:742-749.

9. Jaski BE, Lingle RJ, Reardon LC, et al. Left ventricular assist device as a bridge to patient and myocardial recovery. Prog Cardiovasc Dis 2000;43:5-18.

10. Dandel $M$, Weng $Y$, Siniawski $H$, et al. Long-term results in patients with idiopathic dilated cardiomyopathy after weaning from left ventricular assist devices. Circulation 2005;112:137-I45.

11. Muller J, Wallukat $G$, Weng YG, et al. Weaning from mechanical cardiac support in patients with idiopathic dilated cardiomyopathy. Circulation 1997;96:542-549.

12. de Jonge $\mathrm{N}$, van Wichen DF, Schipper ME, et al. Left ventricular assist device in end-stage heart failure: persistence of structural myocyte damage after unloading. An immunohistochemical analysis of the contractile myofilaments. J Am Coll Cardiol 2002;39:963-969.

13. Goldsmith EC, Borg TK. The dynamic interaction of the extracellular matrix in cardiac remodeling. J Card Fail 2002;8:S314-S318.

14. Stamenkovic I. Extracellular matrix remodelling: the role of matrix metalloproteinases. J Pathol 2003;200:448-464.

15. Bruggink $A H$, van Oosterhout $M F$, de Jonge $N$, et al. Reverse remodeling of the myocardial extracellular matrix after prolonged left ventricular assist device support follows a biphasic pattern. J Heart Lung Transplant 2006;25:1091-1098.

16. Stack MS, Moser TL, Pizzo SV. Binding of human plasminogen to basement-membrane (type IV) collagen. Biochem J 1992;284(Part 1):103-108.

17. Cleutjens JP. The role of matrix metalloproteinases in heart disease Cardiovasc Res 1996;32:816-821.

18. Hilenski LL, Terracio L, Borg TK. Myofibrillar and cytoskeletal assembly in neonatal rat cardiac myocytes cultured on laminin and collagen. Cell Tissue Res 1991;264:577-587.

19. Kim H, Yoon CS, Rah B. Expression of extracellular matrix components fibronectin and laminin in the human fetal heart. Cell Struct Funct 1999:24:19-26.

20. Farhadian F, Contard F, Corbier A, et al. Fibronectin expression during physiological and pathological cardiac growth. J Mol Cell Cardiol 1995;27:981-990.

21. Strickland S, Reich E, Sherman MI. Plasminogen activator in early embryogenesis: enzyme production by trophoblast and parietal endoderm. Cell 1976;9:231-240.

22. Highsmith RF. Isolation and properties of a plasminogen activator derived from canine vascular tissue. J Biol Chem 1981;256:6788-6795.

23. Reich $R$, Thompson EW, Iwamoto $Y$, et al. Effects of inhibitors of plasminogen activator, serine proteinases, and collagenase IV on the invasion of basement membranes by metastatic cells. Cancer Res 1988;48:3307-3312.

24. Spinale FG, Coker ML, Heung LJ, et al. A matrix metalloproteinase induction/activation system exists in the human left ventricular myocardium and is upregulated in heart failure. Circulation 2000;102:1944-1949.

25. Li YY, Feldman AM, Sun Y, et al. Differential expression of tissue inhibitors of metalloproteinases in the failing human heart. Circulation 1998:98:1728-1734.

26. Van Hoffen E, Van Wichen D, Stuij I, et al. In situ expression of cytokines in human heart allografts. Am J Pathol 1996;149:1991-2003.

27. Van Hoffen E, Van Wichen DF, Leemans JC, et al. T cell apoptosis in human heart allografts: association with lack of co-stimulation? Am J Pathol 1998;153:1813-1824.

28. Havenith MG, Cleutjens JP, Beek C, et al. Human specific anti-type IV collagen monoclonal antibodies, characterization and immunohistochemical application. Histochemistry 1987;87:123-128.

29. Rouet-Benzineb P, Buhler JM, Dreyfus $P$, et al. Altered balance between matrix gelatinases (MMP-2 and MMP-9) and their tissue inhibitors in human dilated cardiomyopathy: potential role of MMP-9 in myosinheavy chain degradation. Eur J Heart Fail 1999;1:337-352.

30. Deng MC, Edwards LB, Hertz Ml, et al. Mechanical circulatory support device database of the International Society for Heart and Lung Transplantation: third annual report-2005. J Heart Lung Transplant 2005;24:1182-1187.

31. Burkhoff $D$, Holmes JW, Madigan J, et al. Left ventricular assist deviceinduced reverse ventricular remodeling. Prog Cardiovasc Dis 2000;43:19-26.

32. Spinale FG, Zellner JL, Johnson WS, et al. Cellular and extracellular remodeling with the development and recovery from tachycardiainduced cardiomyopathy: changes in fibrillar collagen, myocyte adhesion capacity and proteoglycans. J Mol Cell Cardiol 1996;28: 1591-1608.

33. Falk V, Soccal PM, Grunenfelder J, et al. Regulation of matrix metalloproteinases and effect of MMP-inhibition in heart transplant related reperfusion injury. Eur J Cardiothorac Surg 2002;22:53-58.

34. Hayashi T, Shimomura H, Terasaki $F$, et al. Collagen subtypes and matrix metalloproteinase in idiopathic restrictive cardiomyopathy. Int J Cardiol 1998;64:109-116.

35. Spinale FG. Matrix metalloproteinases: regulation and dysregulation in the failing heart. Circ Res 2002;90:520-530.

36. Li YY, Feng Y, McTiernan CF, et al. Downregulation of matrix metalloproteinases and reduction in collagen damage in the failing human heart after support with left ventricular assist devices. Circulation 2001;104:1147-1152.

37. Klotz S, Foronjy RF, Dickstein ML, et al. Mechanical unloading during left ventricular assist device support increases left ventricular collagen cross-linking and myocardial stiffness. Circulation 2005;112:364-374.

38. Jo Y, Yeon J, Kim HJ, et al. Analysis of tissue inhibitor of metalloproteinases-2 effect on pro-matrix metalloproteinase-2 activation by membrane-type 1 matrix metalloproteinase using baculovirus/insect-cell expression system. Biochem J 2000;345(Part 3): 511-519.

39. Thomas CV, Coker ML, Zellner JL, et al. Increased matrix metalloproteinase activity and selective upregulation in LV myocardium from patients with end-stage dilated cardiomyopathy. Circulation 1998;97:1708-1715.

40. Reinhardt D, Sigusch HH, Hensse J, et al. Cardiac remodelling in end stage heart failure: upregulation of matrix metalloproteinase (MMP) irrespective of the underlying disease, and evidence for a direct inhibitory effect of ACE inhibitors on MMP. Heart 2002;88:525-530.

41. Masure $S$, Proost $P$, Van Damme J, et al. Purification and identification of $91-\mathrm{kDa}$ neutrophil gelatinase. Release by the activating peptide interleukin-8. Eur J Biochem 1991;198:391-398.

42. Descamps FJ, Martens E, Opdenakker G. Analysis of gelatinases in complex biological fluids and tissue extracts. Lab Invest 2002;82:1607-1608. 